\title{
Correction to: Maternal Progesterone Treatment Reduces Maternal Inflammation-Induced Fetal Brain Injury in a Mouse Model of Preterm Birth
}

\author{
Yuval Ginsberg $^{1} \cdot$ Ola Gutzeit $^{1} \cdot$ Salim Hadad ${ }^{1} \cdot$ Michael Y. Divon $^{2} \cdot$ Nizar Khatib $^{1} \cdot$ Ofer Fainaru $^{1} \cdot$ Zeev Weiner $^{1}$. \\ Ron Beloosesky ${ }^{1}$
}

Published online: 23 September 2020

(C) Society for Reproductive Investigation 2020

Correction to: Reproductive Sciences. https://doi.org/10.1007/s43032-020-00272-w

This article was corrected to update the spelling of author Ola Gutzeit's name.

Publisher's Note Springer Nature remains neutral with regard to jurisdictional claims in published maps and institutional affiliations.

Yuval Ginsberg and Ola Gutzeit contributed equally to this work.

This study was presented at the 38th Annual Meeting of the Society for Maternal-Fetal Medicine (SMFM).

The online version of the original article can be found at https://doi.org/ 10.1007/s43032-020-00272-w

Yuval Ginsberg

y_ginsberg@rambam.health.gov.il

1 Department of Obstetrics and Gynecology, Rambam Health Care, Campus, 8 Ha'alya St, 38302 Haifa, Israel

2 Department of Obstetrics \& Gynecology, Division of Maternal-Fetal Medicine, Lenox Hill Hospital, Northwell Health, New York

City, NY, USA 